\title{
Diameter of random spanning trees in a given graph
}

\author{
Fan Chung * Paul Horn ${ }^{\dagger} \quad$ Linyuan Lu ${ }^{\ddagger}$
}

November 11, 2010

\begin{abstract}
Motivated by the observation that the sparse tree-like subgraphs in a small world graph have large diameter, we analyze random spanning trees in a given host graph. We show that the diameter of a random spanning tree of a given host graph $G$ is between $c \sqrt{n}$ and $c^{\prime} \sqrt{n} \log n$ with high probability., where $c$ and $c^{\prime}$ depend on the spectral gap of $G$ and the ratio of the moments of the degree sequence. For the special case of regular graphs, this result improves the previous lower bound by Aldous by a factor of $\log n$.
\end{abstract}

\section{Introduction}

Many information networks or social networks have very small diameters, as dictated by the so-called "small world phenomenon". In a recent study by Liben-Nowell and Kleinberg [8] on the cascading effect in complex graphs, it was observed that the tree-like subgraphs based on chain-letter data have relatively large diameters. Of special interests are the structure and shape of these tree-like graphs, which was described to be "surprisingly deep and narrow". Questions were raised in the attempt to understanding such sparse graphs that are formed from information propagation in real-world graphs.

A sparse subgraph naturally can have very different behavior from its host graph. It is of interest to understand the connections between a graph and its subgraphs. Numerous questions arise. What are the crucial invariants of the host graph which can be used to control its subgraphs? Under what conditions of these invariants can we predict the behavior of subgraphs? In particular, can the large diameter of tree-like subgraphs, observed by Liben-Nowell and Kleinberg, be quantitatively and rigorously validated? In this paper, we will

\footnotetext{
*University of California, San Diego, fan@math.sc.edu. Research supported in part by ONR MURI N000140810747, and AF/SUB 552082.

$\dagger$ University of California, San Diego, phorn@math.ucsd.edu.

¥University of South Carolina, lu@math.sc.edu. This author was supported in part by NSF grant DMS 0701111 and DMS 1000475.
} 
address this question by considering random spanning trees in a given host graph.

We will analyze the diameter of random spanning trees in a given host graph by using and extending techniques originating in the study of random graphs and random walks on graphs. We use a variant of random walks, called truncated random walks, which are particularly effective in dealing with general graphs with uneven degree distributions (see Section 4 for details). We will show that, under some appropriate conditions, a random spanning tree of a given graph has diameter of order $\sqrt{n}$ (which will be clarified later). This result improves the previous diameter lower bound established by Aldous for regular graphs. In addition, this result also supports the observations made in [8]. Namely, one of the chain-letter trees in [8] with about $n=10,000$ nodes have width about 80 and diameter about 120, both of which are approximately of order $\sqrt{n}$.

To state our result rigorously, we will need definitions. In Section 2, we will state the main theorems and discuss examples and conditions. In Section 3, we mention a number of useful facts on the spectrum of the Laplacian, random walks, and spanning trees. In Section 4, we the method of using random walks to generate a uniform spanning tree in a host graph. In Section 5, we will prove the lower bound for the diameter of a random spanning tree and give an upper bound in Section 6 .

\section{Statements of the main theorems}

A spanning tree $T$ of a connected graph $G$ is an acyclic, connected subgraph of $G$. The diameter of a connected spanning subgraph is always at least the diameter of $G$. However, the diameter of a spanning tree may be much larger than the diameter of the graph. The case that the host graph $G$ is the complete graph $K_{n}$ is well-studied in the literature. The number of spanning trees of $K_{n}$ is $n^{n-2}$ by Cayley's theorem. Rényi and Szekeres [11] showed that the diameter of a uniformly random spanning tree in $K_{n}$ is of order $\sqrt{n}$, in contrast to the fact that the diameter of $K_{n}$ is 1 . At the other extreme, if the underlying host graph is, itself, a tree then there is only one spanning tree and the diameter does not change at all.

Motivated by these examples, we consider the general question of determining the diameter of a random spanning tree for any given host graph. Various host graphs can have diverse behavior and structures. Nevertheless, we wish to examine crucial parameters of the host graph that can effect the diameter of random spanning trees.

Previously Aldous [1] proved that in a regular graph $G$ with spectral bound $\sigma$ (which will be defined later), the expected diameter of a uniformly random spanning tree $T$ of $G$, denoted by $\operatorname{diam}(T)$, has expected value satisfying

$$
\frac{c \sigma \sqrt{n}}{\log n} \leq \mathbb{E}(\operatorname{diam}(T)) \leq \frac{c \sqrt{n} \log n}{\sqrt{\sigma}}
$$

for some absolute constant $c$, where here (and throughout this paper); where 
$\log$ refers to the natural logarithm.

We partially improve Aldous' result as follows:

Theorem 1. For a d-regular graph $G$ on $n$ vertices with spectral gap $\sigma$, a spanning tree $T$ of $G$ has expected value satisfying

$$
c \sqrt{n} \leq \mathbb{E}(\operatorname{diam}(T)) \leq c^{\prime} \frac{\sqrt{n} \log n}{\sqrt{\log (1 / \sigma)}}
$$

for some absolute constants $c$ and $c^{\prime}$, provided that $d \gg \frac{\log ^{2} n}{\log ^{2} \sigma}$.

Theorem 1 is an immediate consequence of the following result for general graphs.

Theorem 2. Suppose $G$ is a connected graph on $n$ vertices, with average degree $d$, minimum degree $\delta$, and second-order average degree $\tilde{d}=\sum_{v} d_{v}^{2} / \sum_{u} d_{u}$, and $\epsilon>0$ is fixed. Suppose the average degree satisfies

$$
d \gg \frac{\log ^{2} n}{\log ^{2} \sigma} .
$$

Then with probability $1-\epsilon$, the diameter $\operatorname{diam}(T)$ of a random spanning tree $T$ in $G$ satisfies

$$
c_{1} \sqrt{n} \leq \operatorname{diam}(T) \leq c_{2} \sqrt{n} \log n,
$$

where $c_{1}$ and $c_{2}$ are constants depending on $\epsilon, d, \delta, \tilde{d}, \sigma$, defined as follows:

$$
c_{1}=(1-\epsilon) \sqrt{\frac{\epsilon d}{\tilde{d}}} .
$$

and

$$
c_{2}=\frac{10}{\epsilon} \sqrt{\frac{d}{\delta \log (1 / \sigma)}} .
$$

Remark 1: While the conditions in Theorem 2 look technical, they are derived from the proofs in Sections 4 and 5. It should be noted that the condition (1) is really a condition on both $d$ and $\sigma$. The smaller the spectral gap $\sigma$ is, the smaller $d$ is allowed to be. For example, (1) is satisfied for any graph with the average degree is at least $\log ^{2} n$, and $\sigma=o(1)$.

Remark 2: The conditions in Theorem 2 are satisfied if

$$
\sigma=o\left(\frac{\log n}{\sqrt{d}}\right) .
$$

For random $d$-regular graphs, it is known that $\sigma$ is about $\frac{2}{\sqrt{d}}$ with high probability. Thus random $d$-regular graphs, with $d$ of order at least $\log ^{2} n$, easily satisfy the conditions (1) and (2). 
Remark 3: Ideally, we would like to let $\epsilon$ be a function of $n$ tending to zero. This can be done and we discuss the necessary requirements after the proof of Theorem 2. The proof is explicit, as opposed to asymptotic, for a graph with fixed average degree $d$, and $\sigma$ (as opposed to the $o(1)$ term in the statement of the theorem), although the expression is somewhat complicated. For simplicity we do not state the technical generalizations here.

Remark 4: For the random graph model $G(\mathbf{w})$ for a given expected degree sequence $\mathbf{w}=\left(w_{1}, w_{2}, \ldots, w_{n}\right)$, as introduced in [4], the probability $p_{i j}$ that there is an edge between $v_{i}$ and $v_{j}$ is proportional to the product $w_{i} w_{j}$ (as well as the loop at $v_{i}$ with probability proportional to $w_{i}^{2}$ ). It has been shown in [6] that $G(\mathbf{w})$ has $\sigma \leq(1+o(1)) \frac{4}{\sqrt{d}}$ with high probability provided that the minimum degree is $\Omega(\log n)$. Theorem 2 implies the diameter of random spanning tree of a graph in $G(\mathbf{w})$ is $\Omega\left(\sqrt{\frac{d}{d} n}\right)$ with high probability, if the average degree is $d=\Omega\left(\left(\frac{\log n}{\log \log n}\right)^{2}\right)$. The upper bound for the diameter is within a multiplicative factor of $O\left(\sqrt{\frac{\tilde{d}}{\delta}} \log n\right)$.

Remark 6: It has been observed that many real-world networks satisfy the socalled power law. We say a family of graphs satisfies a power law with exponent $\beta$ if the degree sequence of the graph satisfies the property that the number of vertices having degree $k$ is asymptotically proportional to $k^{-\beta}$. There are many models being used to capture the behavior of such power law graphs [5], especially for the exponent $\beta$ in the range between 2 and 3. Suppose we use the random graph model $G(\mathbf{w})$ with w satisfying the power law to generate graphs with a power-law degree distribution. The $G(\mathbf{w})$ model is appropriate for this task, so long as the (expected) maximum degree $m$ is no larger than $\sqrt{n}$. (In other words, if the maximum degree exceeds $\sqrt{n}$, then $G(\mathbf{w})$ can only be used to model the subgraph with degree no larger than $\sqrt{n}$.) Generating a random power law graph in such a fashion, and noting that the second average degree is of order $d^{\beta-1} m^{3-\beta}$, we may use the Theorem 2 to study the diameter of random spanning trees in power law graphs. Setting $m=\sqrt{n}$ and using Theorem 2, the diameter of a random spanning tree in such a random power law graph is at least $c n^{(\beta-2) / 4}(\log n)^{(2-\beta) / 2}$ and at most $c^{\prime} \sqrt{n}(\log n)^{3 / 2}$ for some constants $c$ and $c^{\prime}$, with high probability.

\section{Preliminary Spectral Lemmas}

Suppose $G$ is a connected (non-bipartite) graph on vertex set $[n]=\{1,2, \ldots, n\}$. Let $A=\left(a_{i j}\right)$ be the adjacency matrix of $G$ defined by

$$
a_{i j}= \begin{cases}1 & \text { if } i j \text { is an edge; } \\ 0 & \text { otherwise. }\end{cases}
$$

For $1 \leq i \leq n$ let $d_{i}=\sum_{j} a_{i j}$ be the degree of vertex $i$. Let $\Delta=\max \left(d_{1}, \ldots, d_{n}\right)$ be the maximum degree and $\delta=\min \left(d_{1}, \ldots, d_{n}\right)$ be the minimum degree. For 
each $k$, we define the $k$-th volume, closely related to the $k$-th moment of the degree sequence, of $G$ to be

$$
\operatorname{vol}_{k}(G)=\sum_{i=1}^{n} d_{i}^{k} .
$$

The volume $\operatorname{vol}(G)$ is simply the sum of all degrees, i.e. $\operatorname{vol}(G)=\operatorname{vol}_{1}(G)$.

We define the average degree $d=\frac{1}{n} \operatorname{vol}(G)=\frac{\operatorname{vol}_{1}(G)}{\operatorname{vol}_{0}(G)}$ and the second order average degree $\tilde{d}=\frac{\operatorname{vol}_{2}(G)}{\operatorname{vol}_{1}(G)}$.

Let $D=\operatorname{diag}\left(d_{1}, d_{2}, \ldots, d_{n}\right)$ denote the diagonal degree matrix. The Laplacian matrix is defined as

$$
\mathcal{L}=I-D^{-\frac{1}{2}} A D^{-\frac{1}{2}}
$$

The spectrum of the Laplacian is the sequence of eigenvalues of $\mathcal{L}$ sorted in increasing order.

$$
0=\lambda_{0} \leq \lambda_{1} \leq \cdots \leq \lambda_{n-1}
$$

The first eigenvalue $\lambda_{0}$ is always equal to $0 . \lambda_{1}>0$ if $G$ is connected and $\lambda_{n-1} \leq 2$ with equality holding only if $G$ is bipartite graph.

Let $\sigma=\max \left\{1-\lambda_{1}, \lambda_{n-1}-1\right\}$. Thus $\sigma<1$ if $G$ is connected and nonbipartite. Note that $\sigma$ is closely related to the mixing rate of random walks on $G$.

Let $\alpha_{0}, \alpha_{1}, \ldots, \alpha_{n-1}$ be orthonormal (row) eigenvectors of the Laplacian $\mathcal{L}$, $U=\left(\alpha_{0}^{*}, \alpha_{1}^{*}, \ldots, \alpha_{n-1}^{*}\right)$. Also we define $\Lambda=\operatorname{diag}\left(\lambda_{0}, \ldots, \lambda_{n-1}\right)$. We can write

$$
\mathcal{L}=U \Lambda U^{*}
$$

For $0 \leq i \leq n-1$, we define $\phi_{i}=\alpha_{i} D \alpha_{i}^{*}$. We define $\left(\phi_{0}, \phi_{1}, \ldots, \phi_{n-1}\right)$ to be the degree spectrum. Then we have the following useful facts:

Lemma 1. The degree spectrum satisfies the following properties.

1. $\phi_{0}=\tilde{d}$.

2. For $0 \leq i \leq n-1, \delta \leq \phi_{i} \leq \Delta$.

3. $\sum_{i=0}^{n-1} \phi_{i}=\operatorname{vol}(G)$.

Proof. Note $\phi_{0}=\left(\frac{\sqrt{d_{1}}}{\sqrt{\operatorname{vol}(G)}}, \ldots, \frac{\sqrt{d_{n}}}{\sqrt{\operatorname{vol}(G)}}\right)^{*}$ since $\mathcal{L} \phi_{0}=0$. We have

$$
\begin{aligned}
\phi_{0} & =\alpha_{0} D \alpha_{0}^{*} \\
& =\sum_{i=1}^{n} \frac{\sqrt{d_{i}}}{\sqrt{\operatorname{vol}(G)}} d_{i} \frac{\sqrt{d_{i}}}{\sqrt{\operatorname{vol}(G)}} \\
& =\frac{\sum_{i=1}^{n} d_{i}^{2}}{\operatorname{vol}(G)} \\
& =\tilde{d}
\end{aligned}
$$


We have

$$
\begin{aligned}
\left|\phi_{i}-\frac{\delta+\Delta}{2}\right| & =\left|\alpha_{i} D \alpha_{i}^{*}-\frac{\delta+\Delta}{2}\right| \\
& =\left|\alpha_{i}\left(D-\frac{\delta+\Delta}{2} I\right) \phi_{i}^{*}\right| \\
& \leq\left\|D-\frac{\delta+\Delta}{2} I\right\| \\
& =\frac{\Delta-\delta}{2} .
\end{aligned}
$$

Thus, we have

$$
\delta \leq \phi_{i} \leq \Delta
$$

We also have

$$
\begin{aligned}
\sum_{i} \phi_{i} & =\operatorname{Tr}\left(U^{*} D U\right) \\
& =\operatorname{Tr}(D) \\
& =\operatorname{vol}(G) .
\end{aligned}
$$

Lemma 2. For any integer $j \geq 1$,

$$
\operatorname{Tr}\left(A\left(D^{-1} A\right)^{j-1}\right) \leq \tilde{d}+\sigma^{j}(\operatorname{vol}(G)-\tilde{d}) .
$$

Proof. We have

$$
\begin{aligned}
\operatorname{Tr}\left(A\left(D^{-1} A\right)^{j-1}\right) & =\operatorname{Tr}\left(D\left(D^{-1} A\right)^{j}\right) \\
& =\operatorname{Tr}\left(D\left(D^{-\frac{1}{2}} A D^{-\frac{1}{2}}\right)^{j}\right. \\
& =\operatorname{Tr}\left(D(I-\mathcal{L})^{j}\right) \\
& =\operatorname{Tr}\left(D U(I-\Lambda)^{j} U^{*}\right) \\
& =\operatorname{Tr}\left(U^{*} D U(I-\Lambda)^{j}\right) \\
& =\sum_{i=0}^{n-1} \phi_{i}\left(1-\lambda_{i}\right)^{j} \\
& =\tilde{d}+\sum_{i>0} \phi_{i}\left(1-\lambda_{i}\right)^{j} \\
& \leq \tilde{d}+\sum_{i>0} \phi_{i} \sigma^{j} \\
& =\tilde{d}+(\operatorname{vol}(G)-\tilde{d}) \sigma^{j} .
\end{aligned}
$$

A simple random walk on $G$ is a sequence of vertices $v_{0}, v_{1}, \ldots, v_{k}, \ldots$ with

$$
\mathbb{P}\left(v_{k}=j \mid v_{k-1}=i\right)=p_{i j}= \begin{cases}\frac{1}{d_{i}} & \text { if } i j \in E(G) \\ 0 & \text { otherwise }\end{cases}
$$


for all $k \geq 1$.

The transition matrix $P$ is a $n \times n$ matrix with entries $p_{i j}$ for $1 \leq i, j \leq n$. We can write $P=D^{-1} A$.

A probability distribution over the set of vertices is a row vector $\beta\left(\beta^{*} \in \mathbb{R}^{n}\right)$ satisfying

1. The entries of $\beta$ are non-negative.

2. The $L_{1}$-norm $\|\beta\|_{1}(=\beta \mathbf{1})$ equal to 1 where 1 denotes a column vector with all entries 1.

If $\beta$ is a probability distribution, so is $\beta P$. The stationary distribution is a vector, denoted by $\pi$, satisfying $\pi=\pi P$ and

$$
\pi=\frac{1}{\operatorname{vol}(G)}\left(d_{1}, d_{2}, \ldots, d_{n}\right) .
$$

The eigenvalues of $P$ are $1,1-\lambda_{1}, \ldots, 1-\lambda_{n-1}$, since $P=D^{-\frac{1}{2}}(I-\mathcal{L}) D^{\frac{1}{2}}$. In general, $P$ is not symmetric unless $G$ is regular.

The following lemma concerns the mixing rate of the random walks; similar mixing lemmas are well known, see, eg. [3].

Lemma 3. For any integer $t>0$, any $\alpha \in \mathbb{R}^{n}$, and any two probability distributions $\beta$ and $\gamma$, we have

$$
\left.\left\langle(\beta-\gamma) P^{t}, \alpha D^{-1}\right\rangle \leq \sigma^{t} \|(\beta-\gamma) D^{-1 / 2}\right)\left\|_{2}\right\| \alpha D^{-1 / 2} \|_{2} .
$$

In particular,

$$
\left\|(\beta-\gamma) P^{t} D^{-1 / 2}\right\| \leq \sigma^{t}\left\|(\beta-\gamma) D^{-1 / 2}\right\| .
$$

Proof. Here, we assume all vectors are row vectors (including $\mathbf{1}$ which is the all row vector containing only ones).

Let $\varphi=\frac{1}{\sqrt{\operatorname{vol}(G)}} \mathbf{1}\left(\sqrt{d_{1}}, \ldots, \sqrt{d_{n}}\right)=\operatorname{vol}(G)^{-\frac{1}{2}}\left(D^{\frac{1}{2}}\right)$ denote the (row) eigenvector of $I-\mathcal{L}$ for the eigenvalue 1 . The matrix $(I-\mathcal{L})^{t}-\varphi^{*} \varphi$, which is the projection of $(I-\mathcal{L})^{t}$ to the subspace $\varphi^{\perp}$, has $L_{2}$-norm $\sigma^{t}$. Note that

$$
(\beta-\gamma) D^{-\frac{1}{2}} \varphi^{*}=\frac{1}{\operatorname{vol}(G)}(\beta-\gamma) \mathbf{1}=0 .
$$

We have

$$
\begin{aligned}
\left\langle(\beta-\gamma) P^{t}, D^{-1} \alpha^{*}\right\rangle & =(\beta-\gamma) D^{-\frac{1}{2}}\left[(I-\mathcal{L})^{t}-\varphi^{*} \varphi\right] D^{-\frac{1}{2}} \alpha^{*} \\
& \leq\left\|(\beta-\gamma) D^{-\frac{1}{2}}\right\|_{2} \sigma^{t}\left\|\alpha D^{-\frac{1}{2}}\right\|_{2} .
\end{aligned}
$$

Now we choose $\alpha=(\beta-\gamma) P^{t}$ to obtain (4) as desired.

The mixing rate of the random walks on $G$ measures how fast $\beta P^{t}$ converges to the stationary distribution $\pi$ from an initial distribution $\beta$. We can use the above lemma to show that the distribution $\beta P^{t}$ converges to $\pi$ rapidly if $\sigma$ is strictly less than 1 . 


\section{Random spanning trees generated by random walks}

Assume that we have a fixed graph $G$, and we wish to understand uniformly random spanning trees within this graph $G$. The following so-called groundskeeper algorithm gives a method of generating spanning trees of $G$ using random walks: Start a random walk at a vertex, $v$. When a vertex is visited first time via some edge, we add that edge to our spanning tree. Once the graph is covered, the resulting set of edges form a spanning tree. This gives a map $\Phi$ from random walks to random spanning trees. Aldous [1] and Broder [2] independently show that the groundskeeper algorithm generates a uniformly random spanning tree:

Theorem 3 (Groundskeeper Algorithm). The image of $\Phi$ is uniformly distributed over all spanning trees. It is independent of the choice of initial vertex $v$.

If we pick a random initial vertex with stationary distribution $\pi$, then at any step $t$, the vertex visited by our random walk is distributed according to $\pi$.

Starting a random walk at our uniformly chosen initial vertex, we generate a random spanning tree. We derive, for an integer $g \geq 3$, a $g$-truncated random walk from the random walk defining our spanning tree by stopping the first time our random walker visits a site that he has last visited more than $g-2$ steps in the past. That is, if our random walk is a sequence of vertices $v_{1}, v_{2}, \ldots$, we allow the backtrack step $v_{t+1}=v_{t-i}$ for some $i \leq g-2$. However, if $v_{t+1}=v_{t-i}$ for some $i>g-2$, the random walk stops. Recall that we build our spanning tree by recording the edges $v_{t-1} v_{t}$ the first time $v_{t}$ is visited.

Lemma 4. The probability that a g-truncated random walk stops before or at time $t$ is at most

$$
\frac{(t-g+3)(t-g+2) \tilde{d}}{2 n d}+(t-g+2) \frac{\sigma^{g}}{1-\sigma} .
$$

Proof. When the truncated random walk stops, there exists a closed walk $C=$ $v_{i}, v_{i+1}, \ldots, v_{t}, v_{i+k}=v_{i}$ of length $k \geq g$ for some $0 \leq i \leq t-k+1$. For a fixed $i$ and $k$, the probability $f(i, k)$ for such a closed walk is at most

$$
\begin{aligned}
f(i, k) & \leq \sum_{\text {closed walk: } v_{i}, \ldots, v_{i+k}=v_{i}} \frac{d_{v_{i}}}{\operatorname{vol}(G)} \prod_{j=1}^{k} \frac{1}{d_{v_{i+j-1}}} \\
& =\frac{1}{\operatorname{vol}(G)} \operatorname{Tr}\left(A\left(D^{-1} A\right)^{k-1}\right) \\
& \leq \frac{\tilde{d}}{\operatorname{vol}(G)}+\sigma^{k}\left(1-\frac{\tilde{d}}{\operatorname{vol}(G)}\right) \\
& <\frac{\tilde{d}}{\operatorname{vol}(G)}+\sigma^{k} .
\end{aligned}
$$


By summing up for $i \geq 0, k \geq g$, and $i+k \leq t+1$, we have

$$
\begin{aligned}
\sum_{i=0}^{t-g+1} \sum_{k=g}^{t-i+1} f(i, k) & =\sum_{i=0}^{t-g+1} \sum_{k=g}^{t-i+1} \frac{\tilde{d}}{\operatorname{vol}(G)}+\sigma^{k} \\
& \leq \frac{(t-g+3)(t-g+2)}{2} \frac{\tilde{d}}{\operatorname{vol}(G)}+\sum_{i=0}^{t-g+1} \sum_{k=g}^{\infty} \sigma^{k} \\
& \leq \frac{(t-g+3)(t-g+2))}{2} \frac{\tilde{d}}{\operatorname{vol}(G)}+(t-g+2) \frac{\sigma^{g}}{1-\sigma} .
\end{aligned}
$$

The lemma now follows.

\section{Proving a lower bound for the diameter of a random spanning tree}

In this section we will prove a diameter lower bound for spanning trees of $G$ as stated in inequality (2) of Theorem 2.

Proof of (2); the lower bound of Theorem 2: Let

$$
t=\left\lfloor\sqrt{\epsilon \frac{d}{\tilde{d}}} n\right\rfloor
$$

and

$$
g=\left\lceil\frac{\log \left(\frac{\epsilon(1-\sigma) \sqrt{\delta}}{4 t \sqrt{\tilde{d}}}\right)}{\log (\sigma)}\right\rceil .
$$

Note that $g$ is chosen so that

$$
\frac{\sigma^{g}}{1-\sigma} \leq \frac{\epsilon}{4 t}
$$

We generate a uniformly random spanning tree starting a random walk $\left(v_{i}\right)_{i=1}^{\infty}$ at a vertex chosen according to the stationary distribution. Consider the $g$-truncated random walk derived from that walk. By Lemma 4, the $g$ truncated random walk will survive up to time $t$ with probability at least

$$
\begin{aligned}
1-\frac{(t-g+3)(t-g+2) \tilde{d}}{2 n d}-(t-g+2) \frac{\sigma^{g}}{1-\sigma} & >1-\frac{t^{2} \tilde{d}}{2 n d}-t \frac{\sigma^{g}}{1-\sigma} \\
& >1-\frac{\epsilon}{2}-\frac{\epsilon}{4} \\
& \geq 1-\frac{3 \epsilon}{4} .
\end{aligned}
$$


For $i=1, \ldots, t$, we say $v_{i-1} v_{i}$ is a forward step if $v_{i} \neq v_{j}$ for some $j<i$; we say $v_{i-1} v_{i}$ is a $k$-backward step if $v_{i}=v_{i-k}$ for some $k \leq g-2$ (here, if $v_{i}=v_{i-k}$ for more than one $k \leq g-2$, we call it a $k$-backward step for the smallest such $k)$.

Let $X_{i}=-k$ if $v_{i-1} v_{i}$ is a $k$-backward step and $X_{i}=1$ otherwise. For all $i$, we have

$$
-(g-2) \leq X_{i} \leq 1 .
$$

Let $Y$ be the distance of $v_{0} v_{t}$ in the random spanning tree (determined by the entire random walk) and $X=\sum_{i=1}^{t} X_{i}$. Note that the $X_{i}$ are defined from the underlying random walk and hence are always defined. Conditioning on the event that the truncated random walk survives up to time $t$, however, we have $Y \geq X$. This implies that

$$
\mathbb{P}(Y<X)<\frac{3 \epsilon}{4}
$$

Let $\mathcal{F}_{i}$ be the $\sigma$-algebra generated by $v_{0}, \ldots, v_{i}$. For $i=0, \ldots, t, \mathbb{E}\left(X \mid \mathcal{F}_{i}\right)$ forms a martingale. We would like to establish a Lipschitz condition for this martingale. For $1 \leq i, j \leq t$, it is enough to bound $\left|\mathbb{E}\left(X_{j} \mid \mathcal{F}_{i}\right)-\mathbb{E}\left(X_{j} \mid \mathcal{F}_{i-1}\right)\right|$. For $j<i, X_{j}$ is completely determined by the information on $v_{0}, v_{1}, \ldots, v_{i}$. In this case we have

$$
\mathbb{E}\left(X_{j} \mid \mathcal{F}_{i}\right)=\mathbb{E}\left(X_{j} \mid \mathcal{F}_{i-1}\right) .
$$

For $j \geq i, \mathbb{E}\left(X_{j} \mid \mathcal{F}_{i}\right)$ and $\mathbb{E}\left(X_{j} \mid \mathcal{F}_{i-1}\right)$ are different because $v_{i}$ is exposed. For $i \leq j \leq i+2 g-3$, we apply the trivial bound

$$
\left|\mathbb{E}\left(X_{j} \mid \mathcal{F}_{i}\right)-\mathbb{E}\left(X_{j} \mid \mathcal{F}_{i-1}\right)\right| \leq g-1 .
$$

For $j \geq i+2 g-2, X_{j}$ only depends on $v_{j-g+2}, v_{j-g+3}, \ldots, v_{j+1}$. Note that the random walk at step $i$ only depends on the current position $v_{i}$ and is independent of history position $v_{0}, \ldots, v_{i-1}$. Thus $\mathbb{E}\left(X_{j} \mid v_{j-g+2}\right)$ is independent of $v_{i}$ because of $i<j-g+2$. We use the mixing of our random walk to show that information gained from knowing $v_{i}$ is quickly lost. Let $p$ be the distribution of $v_{i}$ given $v_{i-1}$ and $q$ be the distribution of $v_{i}$ given $v_{i}$ ( $q$ is a singleton distribution). Let $\mathbf{p}^{\prime}$ be the distribution of $v_{j-g+2}$ given $v_{i-1}$ and $\mathbf{q}^{\prime}$ be the distribution of $v_{j-g+2}$ given $v_{i}$. (Note: Here $\mathbf{p}^{\prime}$ is not $\mathbf{p}$ transposed.) Applying Lemma 3 , we have

$$
\left\|\left(\mathbf{p}^{\prime}-\mathbf{q}^{\prime}\right) D^{-1 / 2}\right\| \leq\left\|(\mathbf{p}-\mathbf{q}) D^{-1 / 2}\right\| \sigma^{j-g+2-i} \leq \frac{2}{\sqrt{\delta}} \sigma^{j-g+2-i} .
$$

Therefore,

$$
\begin{aligned}
\left|\mathbb{E}\left(X_{j} \mid \mathcal{F}_{i}\right)-\mathbb{E}\left(X_{j} \mid \mathcal{F}_{i-1}\right)\right| & =\left|\sum_{u=1}^{n}\left(\mathbf{p}_{u}^{\prime}-\mathbf{q}_{u}^{\prime}\right) \mathbb{E}\left(X_{j} \mid v_{j-g+2}=u\right)\right| \\
& \leq\left\|\mathbf{p}^{\prime}-\mathbf{q}^{\prime}\right\|_{1}(g-2) \\
& \leq \sqrt{\operatorname{vol}(G)}\left\|\left(\mathbf{p}^{\prime}-\mathbf{q}^{\prime}\right) D^{-1 / 2}\right\|(g-2) \\
& \leq 2(g-2) \frac{\sqrt{\operatorname{vol}(G)}}{\sqrt{\delta}} \sigma^{j-g+2-i} .
\end{aligned}
$$


We have

$$
\begin{aligned}
\left|\mathbb{E}\left(X \mid \mathcal{F}_{i}\right)-\mathbb{E}\left(X \mid \mathcal{F}_{i-1}\right)\right| & \leq \sum_{j=1}^{t}\left|\mathbb{E}\left(X_{j} \mid \mathcal{F}_{i}\right)-\mathbb{E}\left(X_{j} \mid \mathcal{F}_{i-1}\right)\right| \\
& \leq 2(g-1)^{2}+\sum_{j=i+2 g-2}^{t} 2(g-2) \frac{\sqrt{\operatorname{vol}(G)}}{\sqrt{\delta}} \sigma^{j-g+2-i} \\
& \leq 2(g-1)^{2}+2(g-2) \frac{\sqrt{\operatorname{vol}(G)} \sigma^{g}}{\sqrt{\delta}(1-\sigma)} \\
& \leq 3 g^{2}
\end{aligned}
$$

noting that $g$ has been chosen so that

$$
\frac{\sigma^{g}}{1-\sigma} \leq \frac{\sqrt{\epsilon} \sqrt{\delta}}{4(1-\epsilon) \sqrt{\operatorname{vol}(G)}}
$$

is sufficiently small to make the last inequality hold.

Thus we have established that $\mathbb{E}\left(X \mid \mathcal{F}_{i}\right)$ satisfies the Lipschitz condition with constant $3 g^{2}$. By applying the Azuma-Hoeffding inequality, we have

$$
\mathbb{P}(X-\mathbb{E}(X)<-\alpha)<2 e^{-\frac{\alpha^{2}}{18 g^{4} t}},
$$

for any positive real $\alpha$.

Note that

$$
\begin{aligned}
\mathbb{E}(X) & =\sum_{i=1}^{t} \mathbb{E}\left(X_{i}\right) \\
& =\sum_{i=1}^{t} \sum_{j=1}^{n} \mathbb{E}\left(X_{i} \mid v_{i-1}=j\right) \mathbb{P}\left(v_{i-1}=j\right) \\
& \geq \sum_{i=1}^{t} \sum_{j=1}^{n}\left(\left(1-\frac{g-1}{d_{j}}\right)+\sum_{k=1}^{g-2} \frac{-k}{d_{j}}\right) \frac{d_{j}}{\operatorname{vol}(G)} \\
& =\sum_{i=1}^{t} \sum_{j=1}^{n}\left(1-\frac{g(g-1)}{2 d_{j}}\right) \frac{d_{j}}{\operatorname{vol}(G)} \\
& =\sum_{i=1}^{t}\left(1-\frac{g(g-1) n}{2 \operatorname{vol}(G)}\right) \\
& =\left(1-\frac{g(g-1)}{2 d}\right) t .
\end{aligned}
$$

By choosing $\alpha=\sqrt{18 g^{4} t \log \frac{4}{\epsilon}}$, we have

$$
\mathbb{P}\left(X<\left(1-\frac{g(g-1)}{2 d}\right) t-\sqrt{18 g^{4} t \log \frac{4}{\epsilon}}\right)<\frac{\epsilon}{4} .
$$


Combining our ingredients, we have

$$
\begin{aligned}
& \mathbb{P}\left(Y<\left(1-\frac{g(g-1)}{2 d}\right) t-\sqrt{18 g^{4} t \log \frac{4}{\epsilon}}\right) \\
\leq & \mathbb{P}\left(X<\left(1-\frac{2}{d}\right) t-\sqrt{18 g^{4} t \log \frac{4}{\epsilon}}\right)+\mathbb{P}(Y<X) \\
< & \frac{3 \epsilon}{4}+\frac{\epsilon}{4} \\
= & \epsilon .
\end{aligned}
$$

To complete the proof, it suffices to check that our degree conditions imply that

$$
\left(1-\frac{g(g-1)}{2 d}\right) t-\sqrt{18 g^{4} t \log \frac{4}{\epsilon}}=(1-o(1)) \sqrt{\epsilon \frac{n d}{\tilde{d}}} .
$$

In particular it suffices to check that

$$
\frac{g}{\sqrt{d}}=o(1)
$$

as $g^{4}$ is clearly $o(t)$.

Since

$$
g \leq \frac{\log \left(\frac{4}{\epsilon(1-\sigma)}\right)+\log \left(t \frac{\tilde{d}}{\delta}\right)}{\log (1 / \sigma)}+1
$$

and

$$
\log \left(t \frac{\tilde{d}}{\delta}\right)=\log ((1-\epsilon) \sqrt{\epsilon})+\frac{1}{2} \log \left(\frac{d}{\delta} n\right)
$$

we have $g / \sqrt{d}=o(1)$, since

$$
d \gg \frac{\log ^{2}(n)}{\log ^{2}(1 / \sigma)}
$$

as hypothesized.

Note that this does provide a somewhat complicated, but explicit, bound on the diameter of a uniformly random spanning tree in a given graph; as opposed to simply an asymptotic result: In particular, with probability at least $1-\epsilon$, the diameter of a random spanning tree of a graph on $n$ vertices with minimum degree $\delta$, spectral bound $\sigma$, average degree $d$ and second order average degree $\tilde{d}$ satisfies

$$
\operatorname{diam}(T) \geq\left(1-\frac{g(g-1)}{2 d}-\sqrt{18 g^{4} \sqrt{\frac{\tilde{d}}{\epsilon n d}} \log \frac{4}{\epsilon}}\right) \sqrt{\epsilon \frac{n d}{\tilde{d}}}
$$


where

$$
g=\left\lceil\frac{\log \left(\frac{\epsilon(1-\sigma) \sqrt{\delta}}{4 t \sqrt{\tilde{d}}}\right)}{\log (\sigma)}\right\rceil
$$

is as in the above.

\section{Proving a diameter upper bound}

For the upper bound, we extend the methods in [1] for a general host graph. In particular we provide a generalization of Theorem 15 of the Aldous' paper to give an upper bound in the general degree case.

Here, we let $X_{t}$ denote the position of a random walk at time $t$. We denote by $T_{B}$ the hitting time of a set $B$; that is

$$
T_{B}=\min \left\{t: X_{t} \in B\right\} .
$$

We denote the return time of a set $B$ to be

$$
T_{B}^{+}=\min \left\{t \geq 1: X_{t} \in B\right\} .
$$

(Note that if the random walker does not start in $B, T_{B}=T_{B}^{+}$.)

When considering the probability that our random walk has some property under some number of steps we use the notation $\mathbb{P}_{\rho}$ to denote that we condition on our random walker having initial distribution $\rho$. Likewise, $\mathbb{E}_{\rho}$ denotes expectation conditioning on the initial distribution. If no distribution is given, it is assumed to be starting from the stationary distribution. As a convenient abuse of notation, for a vertex $v, \mathbb{P}_{v}$ denotes starting with the distribution that places weight 1 on $v$.

The first tool is the following, rather standard, mixing lemma.

Lemma 5. For all initial distributions $\rho$ and all $B \subseteq G$,

$$
\mathbb{P}_{\rho}\left(T_{B}>3 \frac{\log n}{\log (1 / \sigma)} \frac{\operatorname{vol}(G)}{\operatorname{vol}(B)}\right) \leq \frac{1}{2}
$$

Proof. We begin by bounding $\left|P^{s}(\rho, B)-\pi(B)\right|$, where $\rho$ is an (arbitrary) initial distribution and $\pi(B)=\frac{\operatorname{vol}(B)}{\operatorname{vol}(G)}$. As before, all vectors are considered to be row vectors. Write

$$
\rho D^{-1 / 2}=\sum_{i} a_{i} \alpha_{i}
$$

where the $\alpha_{i}$ are left eigenvectors of $(I-\mathcal{L})$ corresponding to eigenvalues $\left(1-\lambda_{i}\right)$. Then

$$
a_{0}=\left\langle\rho D^{-1 / 2}, \frac{D^{1 / 2} \mathbf{1}}{\sqrt{\operatorname{vol} G}}\right\rangle=\frac{1}{\sqrt{\operatorname{vol}(G)}} .
$$


Thus

$$
\rho D^{-1 / 2}=\frac{1 D^{1 / 2}}{\operatorname{vol}(G)}+\sum_{i \geq 1} a_{i} \alpha_{i}
$$

Then

$$
\begin{aligned}
\left|P^{s}(\rho, B)-\pi(B)\right| & =\left|\rho P^{s} \chi_{B}-\frac{\mathbf{1} D}{\operatorname{vol}(G)} \chi_{B}\right| \\
& =\left|\left(\rho D^{-1 / 2}(I-\mathcal{L})^{s}-\frac{\mathbf{1} D^{1 / 2}}{\operatorname{vol}(G)}\right) D^{1 / 2} \chi_{B}\right| \\
& =\left|\left(\frac{\mathbf{1} D^{1 / 2}}{\operatorname{vol}(G)}+\sum_{i \geq 1}\left(1-\lambda_{i}\right)^{s} a_{i} \alpha_{i}-\frac{1 D^{1 / 2}}{\operatorname{vol}(G)}\right) D^{1 / 2} \chi_{B}\right| \\
& \leq \sum_{i \geq 1} \sigma^{s}\left|a_{i}\right|\left|\alpha_{i} D^{1 / 2} \chi_{B}\right| \\
& \leq \sigma^{s} \sqrt{n} \operatorname{vol}_{1 / 2}(B) \\
& \leq \sigma^{s} \sqrt{n|B| \operatorname{vol}(B) \mid}
\end{aligned}
$$

where the last step follows from an application of Cauchy-Schwarz inequality.

Let

$$
s=\log \left(\frac{\sqrt{\operatorname{vol}(B)}}{2 \sqrt{n|B|} \operatorname{vol}(G)}\right) / \log (\sigma)
$$

so

$$
\sigma^{s} \sqrt{n|B| \operatorname{vol}(B)}=\frac{\operatorname{vol}(B)}{2 \operatorname{vol}(G)} \text {. }
$$

Fix $t_{i}=i s$, then

$$
\begin{aligned}
\mathbb{P}\left(T_{B}>x\right) & \leq \mathbb{P}\left(X_{t_{1}} \notin B, X_{t_{2}} \notin B, \ldots, X_{t_{x / s}} \notin B\right) \\
& =\mathbb{P}\left(X_{t_{1}} \notin B\right) \mathbb{P}\left(X_{t_{2}} \notin B \mid X_{t_{1}} \notin B\right) \cdots \mathbb{P}\left(X_{t_{x / s}} \notin B \mid X_{t_{j}} \notin B, \forall j<i\right) \\
& \leq\left(1-\frac{\operatorname{vol}(B)}{\operatorname{vol}(G)}+\sqrt{n|B| \operatorname{vol}(B)} \sigma^{s}\right)^{x / s} \\
& \leq\left(1-\frac{\operatorname{vol}(B)}{2 \operatorname{vol}(G)}\right)^{x / s} .
\end{aligned}
$$

Fix $x=2 \log (2) s \frac{\operatorname{vol}(G)}{\operatorname{vol}(B)}$ and it is easy to check that

$$
\mathbb{P}\left(T_{B}>x\right) \leq \frac{1}{2}
$$

In all, we have

$$
x=2 \log (2) \frac{\log \left(\frac{2 \sqrt{n|B| \operatorname{vol}(G)}}{\sqrt{\operatorname{vol}(B)}}\right) \operatorname{vol}(G)}{\log (1 / \sigma) \operatorname{vol}(B)} \leq 3 \frac{\log n}{\log (1 / \sigma)} \frac{\operatorname{vol}(G)}{\operatorname{vol}(B)} .
$$


The following result (and its proof) are due to Aldous [1]. Let $B=\left\{v_{0}, \ldots, v_{c}\right\}$ denote a set of vertices and let $\mathcal{P}_{B}$ denote the event that the path from $v_{0}$ to the root (starting location of our random walker for generating a UST, chosen by the uniform distribution) in a uniform spanning tree starts $v_{0}, v_{1}, \ldots, v_{c}$. Then

\section{Lemma 6.}

$$
\mathbb{P}\left(T_{v_{c}}=\ell \mid \mathcal{P}_{B}\right)=\frac{\mathbb{P}_{v_{c}}\left(T_{B}^{+}>\ell\right)}{\mathbb{E}_{v_{c}}\left(T_{B}^{+}\right)} .
$$

Proof. For $i<c$, we denote the event $\mathcal{D}_{i}$ to be

$$
\mathcal{D}_{i}=\left\{T_{\left\{v_{0}, \ldots, v_{i}\right\}}=T_{v_{i}}, X_{T_{v_{i}}-1}=v_{i-1}\right\} .
$$

In words, $\mathcal{D}_{i}$ is the event that $v_{i}$ is hit before $v_{j}$ for $j<i$, and indeed $v_{i}$ is first hit from $v_{i-1}$, so $\bigcap_{i<c} \mathcal{D}_{i}=\mathcal{P}_{B}$. Then:

$$
\left\{T_{v_{c}}=\ell\right\} \cap \mathcal{P}_{B}=\left\{T_{v_{c}}=\ell=T_{B}\right\} \cap\left(\cap_{i=0}^{c-1} D_{i}\right)
$$

Note that, from the Markov property, it is clear that $\mathbb{P}\left(\bigcap_{i<c} \mathcal{D}_{i} \mid T_{v_{c}}=T_{B}=\ell\right)$ does not depend on $\ell$ (this is the motivation for writing $\mathcal{P}_{B}$ in an obscure way), thus:

$$
\mathbb{P}\left(T_{v_{c}}=\ell \mid \mathcal{P}_{B}\right)=\alpha \mathbb{P}\left(T_{v_{c}}=T_{B}=\ell\right)
$$

for $\ell=0,1, \ldots$ and for some $\alpha$ which (critically) does not depend on $\ell$. We have that:

$$
\begin{aligned}
\mathbb{P}_{v_{c}}\left(T_{B}^{+}>\ell\right) & =\sum_{w} \mathbb{P}_{v_{c}}\left(X_{0}=v_{c}, X_{\ell}=w, X_{i} \notin B \text { for } 1 \leq i \leq \ell\right) \\
& =\frac{1}{\pi\left(v_{c}\right)} \sum_{w} \mathbb{P}_{\pi}\left(X_{0}=v_{c}, X_{\ell}=w, X_{i} \notin B \text { for } 1 \leq i \leq \ell\right) \\
& =\frac{1}{\pi\left(v_{c}\right)} \sum_{w} \mathbb{P}_{\pi}\left(X_{0}=w, X_{\ell}=v_{c}, X_{i} \notin B \text { for } 1 \leq i \leq \ell\right) \\
& =\frac{1}{\pi\left(v_{c}\right)} \mathbb{P}_{\pi}\left(X_{\ell}=v_{c}, X_{i} \notin B \text { for } 1 \leq i \leq \ell\right) \\
& =\frac{1}{\pi\left(v_{c}\right)} \mathbb{P}_{\pi}\left(T_{v_{c}}=T_{B}=\ell\right)
\end{aligned}
$$

with the third to last equality following from time reversal for the stationary Markov chain. This implies:

$$
\mathbb{P}_{\pi}\left(T_{v_{c}}=\ell \mid \mathcal{P}_{B}\right)=\alpha \pi\left(v_{c}\right) \mathbb{P}_{v_{c}}\left(T_{B}^{+}>\ell\right)
$$

Note finally, then that

$$
1=\sum_{\ell=0}^{\infty} \mathbb{P}_{\pi}\left(T_{v_{c}}=\ell \mid \mathcal{P}_{B}\right)=\sum_{\ell=0}^{\infty} \alpha \pi\left(v_{c}\right) \mathbb{P}_{v_{c}}\left(T_{B}^{+}>\ell\right)=\frac{\alpha \pi\left(v_{c}\right)}{\mathbb{E}_{v_{c}}\left(T_{B}^{+}\right)}
$$

so $\alpha \pi\left(v_{c}\right)=\mathbb{E}_{v_{c}}\left(T_{B}^{+}\right)$, implying the result. 
One can observe that, actually, while the normalizing constant is easy to compute, the exact value is unnecessary for the proof of the upper bound itself.

We now prove the upper bound, establishing (3) in Theorem 2; whose proof mimics that of Aldous.

Proof of (3); the upper bound from Theorem 2: Let us start our random walk from the stationary distribution (unless explicitly noted, all probabilities related with the random walk which generates the spanning tree are taken to start with $\pi)$.

We begin by fixing a path $v_{0}, v_{1}, \ldots, v_{c}$ in our graph; and $B$ be the set $\left\{v_{0}, \ldots, v_{c}\right\}$. As above, $\mathcal{P}_{B}$ will denote the event that the path from $v_{0}$ to the root (that is, the starting location of our random walk, $X_{0}$ ) in our uniform spanning tree starts out along the path $v_{0}, \ldots, v_{c}$. If we let

$$
s=\left\lceil\frac{3}{\log (1 / \sigma)} \frac{\operatorname{vol}(G)}{(c+1) \delta}\right\rceil \geq \frac{3}{\log (1 / \sigma)} \frac{\operatorname{vol}(G)}{\operatorname{vol}(B)} \log n
$$

then, by iterating Lemma 5 we have that

$$
\mathbb{P}_{v_{c}}\left(T_{B}^{+}>j s\right) \leq \frac{1}{2^{j-1}} \mathbb{P}_{v_{c}}\left(T_{B}^{+}>s\right) .
$$

We are now in the position to apply Lemma 6 to both sides; note that the normalizing constant will cancel and we are left with:

$$
\mathbb{P}\left(T_{v_{c}}=j s \mid \mathcal{P}_{B}\right) \leq(1 / 2)^{j-1} \mathbb{P}\left(T_{v_{c}}=s \mid \mathcal{P}_{B}\right) \leq(1 / 2)^{j-1} s^{-1} .
$$

where the last inequality follows from the fact that the right hand side of (5) in Lemma 6 is decreasing with $l$ and hence the left hand side must decrease as well. This monotonicity property, and summing gives:

$$
\mathbb{P}\left(j s \leq T_{v_{c}} \leq(j+1) s \mid \mathcal{P}_{B}\right) \leq(1 / 2)^{j-1} .
$$

Further summing gives

$$
\mathbb{P}\left(j s \leq T_{v_{c}} \mid \mathcal{P}_{B}\right) \leq(1 / 2)^{j-2} .
$$

If $\mathcal{P}_{B}$ occurs, then naturally we have that the distance from $v_{0}$ to the root, $X_{0}$, satisfies

$$
X S d\left(X_{0}, v_{0}\right) \leq d\left(X_{0}, v_{c}\right)+c \leq T_{v_{c}}+c .
$$

We also clearly have that if $d\left(X_{0}, v_{0}\right)>c$, then $\mathcal{P}_{B}$ occurs for some unique path $v_{0}, \ldots, v_{c}$. Thus:

$$
\mathbb{P}\left(d\left(X_{0}, v_{0}\right)>c+j s\right) \leq(1 / 2)^{j-2} .
$$

Clearly $\operatorname{diam}(T) / 2 \leq \max _{v} d\left(X_{0}, v\right) ;$ so

$$
\mathbb{P}(\operatorname{diam}(T) / 2>c+j s) \leq n(1 / 2)^{j-2}
$$


This gives us

$$
\mathbb{E}(\operatorname{diam}(T)) \leq 2 c+3 s \log n \leq 2 c+\frac{3 \operatorname{vol}(G)}{c \log (1 / \sigma) \delta} \log ^{2} n,
$$

with the second inequality coming from the definition of $S$. These terms are the same order of magnitude when setting $c=\sqrt{\frac{\operatorname{vol} G}{\delta \log (1 / \sigma)}} \log n$; giving the desired bound. To establish the bound in the form stated in (3), simply apply Markov's inequality,

Note that by minimizing

$$
2 c+\frac{3 \operatorname{vol}(G)}{c \log (1 / \sigma) \delta} \log ^{2} n
$$

we actually get that

$$
\mathbb{E}(\operatorname{diam}(T)) \leq 2 \sqrt{6 \frac{\operatorname{vol}(G)}{\delta \log (1 / \sigma)}} \log n .
$$

\section{References}

[1] D. Aldous, The Random Walk construction of Uniform Spanning Trees, SIAM J. Discrete Math. 3 (1990), 450-465.

[2] A. Broder, Generating random spanning trees, Symp. foundations of Computer Sci., Institute for Electrical and Electronic Engineers, New York 442447.

[3] F. Chung, Spectral Graph Theory, AMS Publications, 1997.

[4] F. Chung and L. Lu, Connected components in a random graph with given degree sequences, Annals of Combinatorics 6, (2002), 125-145.

[5] F. Chung and L. Lu, Complex Networks and Graphs, CBMS Lecture Series, No. 107, AMS Publications, 2006.

[6] F. Chung, L. Lu and V. Vu, The spectra of random graphs with given expected degrees, Proceedings of National Academy of Sciences, 100, no. 11, (2003), 63 13-6318.

[7] F. Kirchhoff, Über die Auflösung der Gleichungen, auf welche man bei der Undersuchung der linearen Verteilung galvanisher Str" ome gef" uhrt wird, Ann. Phys. chem. 72 (1847) 497-508.

[8] D. Liben-Nowell, J. Kleinberg. Tracing Information Flow on a Global Scale Using Internet Chain-Letter Data. Proc. National Academy of Sciences, (2008), 105(12),4633-4638. 
[9] R. Lyons, Asymptotic enumeration of spanning trees. Combin. Probab. Comput. 14 (2005), no. 4, 491-522.

[10] R. Pemantle, Uniform random spanning trees, Topics in contemporary probability and its applications, J. L. Snell, editor, 1 - 54. CRC Press: Boca Raton (1994).

[11] A. Rényi and G. Szekeres, On the height of trees, J. Austral. Math. Soc., 7 (1967), pp. 497-507. 